SCIENTIFIC LETTER

\title{
Reduced 24 hour ambulatory blood pressure and abnormal heart rate variability in patients with dysorexia nervosa
}

\author{
N D Cong, T Saikawa, R Ogawa, M Hara, N Takahashi, T Sakata
}

Heart 2004;90:563-564. doi: 10.1136/hrt.2003.024356

$\mathrm{D}$ ysorexia nervosa (DN) includes two types of eating disorder: anorexia nervosa (AN) and bulimia nervosa (BN). AN is a syndrome characterised by changes in eating habits, distortions of body shape, and a refusal to maintain even minimally normal body weight. $\mathrm{BN}$ is a syndrome encompassing repeated episodes of binge eating, followed by inappropriate compensatory behaviours such as self induced vomiting, misuse of laxatives and diuretics, fasting or excessive exercise. Both AN and BN predominantly affect young females, in whom the prevalence is approximately 10 times higher than in males, and both are important social health concerns in developed countries. ${ }^{1}$

The following study was designed to explore the underlying autonomic dysfunction in DN; six patients with AN, eight with BN and 11 healthy subjects were assessed. The circadian changes in blood pressure (BP), heart rate (HR), and heart rate variability (HRV) of the patients were measured.

\section{METHODS}

Twenty four hour ambulatory BP and HR were recorded using a portable multibiomedical recorder (TM-2425, A\&D Co, Tokyo, Japan). The measurements of BP and HR were repeated every 30 minutes in the waking period from 6 am to $11 \mathrm{pm}$ and every 60 minutes during sleep from $11 \mathrm{pm}$ to 6 am. Systolic and diastolic BP were measured by both Korotkoff and oscillometric methods. Frequency domain indices of HRV were simultaneously calculated from the data. Ranges of $0.05-0.15 \mathrm{~Hz}$ and $0.15-0.40 \mathrm{~Hz}$ were defined as low frequency (LF) and high frequency (HF) component ranges, respectively. The power HF component was assumed as an index of parasympathetic activity, and the ratio of the powers of LF and HF (LF/HF) as an index of sympathetic activity. $^{2}$

\section{RESULTS}

There was no significant difference in age, height or body weight between the $\mathrm{BN}$ and control groups (AN $33.6 \mathrm{~kg}, \mathrm{BN}$ $47.6 \mathrm{~kg}$, control $50.9 \mathrm{~kg}$, respectively), or between the body mass indices (BMI) of $\mathrm{BN}$ and control groups (AN $13.9 \mathrm{~kg}$ / $\mathrm{m}^{2}$, BN $19.1 \mathrm{~kg} / \mathrm{m}^{2}, 20.3 \mathrm{~kg} / \mathrm{m}^{2}$, respectively). Both body weight and BMI in the AN group were lower than in the BN and control groups. As shown in table $1, \mathrm{AN}$ and BN patients exhibited lower mean 24 hour systolic BP than controls. The mean 24 hour diastolic BP was lower than the controls in the AN group but not in the BN group. The mean 24 hour HR revealed no significant difference among $\mathrm{AN}, \mathrm{BN}$, and control groups (table 1). Both systolic and diastolic BP during wakefulness were lower in $\mathrm{AN}$ and $\mathrm{BN}$ groups than in controls (table 1). The incidence of the non-dipper BP pattern in the $\mathrm{AN}$ and $\mathrm{BN}$ groups was $66.7 \%$ and $62.5 \%$, respectively, higher than in the control group $(9.1 \%)(p=0.0276$ and 0.0408 , respectively).

The power of $\mathrm{HF}$ in $\mathrm{AN}$ and $\mathrm{BN}$ patients was increased significantly by $10.3 \%$ and $12.5 \%$, respectively, compared to controls $(\mathrm{p}=0.0462$ and 0.0163 , respectively). This in turn resulted in a significantly lower LF/HF ratio in the 24 hour analysis in AN and BN groups compared to controls. The HF power in $\mathrm{AN}$ and $\mathrm{BN}$ groups remained higher than controls during waking $(\mathrm{p}=0.0492$ and 0.0091 , respectively) and during sleep $(\mathrm{p}=0.0244$ and 0.0102 , respectively $)$. Consequently, the LF/HF ratio in $\mathrm{AN}$ and $\mathrm{BN}$ groups was

Abbreviations: AN, anorexia nervosa; BMl, body mass index; $\mathrm{BN}$, bulimia nervosa; BP, blood pressure; DN, dysorexia nervosa; HR, heart rate; HRV, heart rate variability; HF, high frequency; LF, low frequency

\begin{tabular}{|c|c|c|c|}
\hline & $\mathrm{AN}(n=6)$ & $\mathrm{BN}(n=8)$ & $\mathrm{CN}(\mathrm{n}=11)$ \\
\hline \multicolumn{4}{|l|}{ Whole day (24 hours) } \\
\hline $\mathrm{SBP}(\mathrm{mm} \mathrm{Hg})$ & $91.0(8.4)^{* *}$ & $97.3(6.8)^{\star \star *}$ & $107.9(10.9)$ \\
\hline $\mathrm{DBP}(\mathrm{mm} \mathrm{Hg})$ & $54.6(4.9)^{* *}$ & $58.5(2.9)$ & $62.7(5.3)$ \\
\hline HR (bpm) & $63.3(5.2)$ & $64.8(10.5)$ & $68.9(6.9)$ \\
\hline \multicolumn{4}{|c|}{ Waking period $(6 \mathrm{am}-11 \mathrm{pm})$} \\
\hline $\mathrm{SBP}(\mathrm{mm} \mathrm{Ha})$ & $92.5(8.3)^{* *}$ & $100.8(8.7)^{* \star *}$ & $113.4(11.7)$ \\
\hline $\mathrm{DBP}(\mathrm{mm} \mathrm{Hg})$ & $56.9(5.0)^{* *}$ & $60.8(4.6)^{* \star *}$ & $66.4(5.6)$ \\
\hline HR (bpm) & $68.0(5.2)$ & $69.6(10.5)$ & $74.0(7.1)$ \\
\hline \multicolumn{4}{|c|}{ Sleeping period ( $11 \mathrm{pm}-6 \mathrm{am}$ ) } \\
\hline $\mathrm{SBP}(\mathrm{mm} \mathrm{Hg})$ & $87.9(9.9)$ & $90.3(5.6)$ & $96.8(12.4)$ \\
\hline $\mathrm{DBP}(\mathrm{mm} \mathrm{Hg})$ & 49.8 (5.5) & $53.9(3.8)$ & $55.3(6.2)$ \\
\hline $\mathrm{HR}$ (bpm) & $53.9(7.5)$ & $55.4(12.0)$ & $58.7(7.7)$ \\
\hline Dipper/non-dipper & $2 / 4^{*}$ & $3 / 5^{* \star *}$ & $10 / 1$ \\
\hline
\end{tabular}

Data are presented as mean (SD)

DBP, diastolic blood pressure; HR, heart rate; SBP, systolic blood pressure. ${ }^{*} \mathrm{p}<0.05 \vee \mathrm{CN}$ group; ${ }^{* *} \mathrm{p}<0.01 \vee \mathrm{CN}$ group; ${ }^{* * *} \mathrm{p}<0.05 \vee \mathrm{CN}$ group. 
concomitantly lower than in controls during both waking and sleeping, respectively.

\section{DISCUSSION}

Analysis of BP and HR in AN patients is confounded by the extremely low body weight of the subjects which, as shown in the present study, inevitably induces distinct neuroendocrinological modulation of BP and HR via mechanisms such as altered adrenal and thyroid function. Thus, it is possible that the observed changes in BP and HR do not reflect the underlying mechanism(s) of AN but rather are secondary to such neuroendocrinological modulation. To investigate this, we simultaneously analysed $\mathrm{BN}$ patients and found that patients with both AN and BN exhibited similar modulation of BP and HR regardless of the differences in body weight and BMI between the two conditions.

The 24 hour ambulatory BP was reduced in both AN and $\mathrm{BN}$ groups and revealed a higher incidence of the non-dipper BP pattern in BN $(62.5 \%)$ and AN (66.7\%) than in controls $(9.1 \%)$. No significant differences in the incidence of the nondipper BP pattern or in indices reflecting autonomic nerve activity were noted between AN and BN groups, although the BMI was significantly different between the two. In addition, both $\mathrm{AN}$ and BN groups were associated with increased HF power and a decreased LF/HF ratio throughout the 24 hour period of wakefulness and sleep. The results were consistent with lower plasma concentrations of noradrenaline (norepinephrine) and lower $\mathrm{BP}$ in $\mathrm{AN}$ and $\mathrm{BN}$ groups than in controls ${ }^{3}{ }^{4}$ together with a rapid response to the infusion of isoproterenol. ${ }^{3}$ Thus, the low LF/HF and higher incidence of non-dipper BP pattern in AN and BN suggest reduced activity of the sympathetic nervous system in these patients. ${ }^{5}$

In conclusion, we have shown the presence of abnormalities in BP patterns in both AN and BN patients characterised by increased incidence of the non-dipper BP pattern, low BP during wakefulness, and disturbances of the autonomic nervous system with increased parasympathetic and decreased sympathetic activity throughout the 24 hour period. The results suggest the dysfunction of the hypothalamus in DN patients may play an important contributory role to this abnormal BP dynamic pattern and enhance our understanding of autonomic cardiac regulation in AN and $\mathrm{BN}$.

\section{Authors' affiliations}

N D Cong, Department of Medicine, Hanoi, Vietnam

T Saikawa, Department of Cardiovascular Science, Division of Laboratory Science, School of Medicine, Oita Medical University, Hasama, Oita, Japan

R Ogawa, M Hara, N Takahashi, T Sakata, Department of Medicine, Internal Medicine I, School of Medicine, Oita Medical University

Correspondence to: Professor Tetsunori Saikawa, Department of Cardiovascular Science, School of Medicine, Oita Medical University, Hasama, Oita, 879-5593, Japan; saikawa@oita-med.ac.jp

Accepted 22 August 2003

\section{REFERENCES}

1 Hay $\mathbf{P}$. The epidemiology of eating disorder behaviors: an Australian community-based survey. Int J Eat Disorder 1998:23:371-82.

2 Cerutti S, Bianchi AM, Mainardi LT. Spectral analysis of the heart rate variability signal. In: Malik M, Camm AJ, eds. Heart rate variability. Armonk, New York: Futura Publishing Co, Inc, 1995:63-74.

3 George DT, Kaye WH, Goldstein DS, et al. Altered norepinephrine regulation in bulimia: effects of pharmacological challenge with isoproterenol. Psychiat Res 1990;33:1-10.

4 Pirke KM. Central and peripheral noradrenaline regulation in eating disorders. Psychiat Res 1996:62:43-9.

5 Licinio J, Wong ML, Gold PW. The hypothalamic-pituitary-adrenal axis in anorexia nervosa. Psychiat Res 1996;62:75-83

\section{IMAGES IN CARDIOLOGY}

\section{Retroperitoneal haemorrhage following thrombolysis}

A 53 year old man attended the accident and emergency department following a four hour history of severe left sided chest pain radiating through to his back and his left arm. ECG on admission showed $3 \mathrm{~mm}$ ST segment elevation in the inferior leads and he was thrombolysed using tenecteplase with subsequent electrocardiographic evidence of coronary reperfusion.

On the second day following admission he developed progressive lower right sided back pain radiating to his hip and groin. On examination there was bruising but no swelling around his groin. The right hip was held in flexion because of discomfort, movement was limited, and he reported parasthesia over the anterior thigh. The haemoglobin had dropped to $11.8 \mathrm{~g} / \mathrm{dl}$. Aspirin and post-thrombolysis heparin were stopped. An urgent computed tomographic scan confirmed an enlarged right psoas muscle which was approximately twice the diameter of the left (panel). This patient was seen by the surgical team and he was managed conservatively. Over the next three days the tenderness and parasthesia resolved and his haemoglobin remained stable.

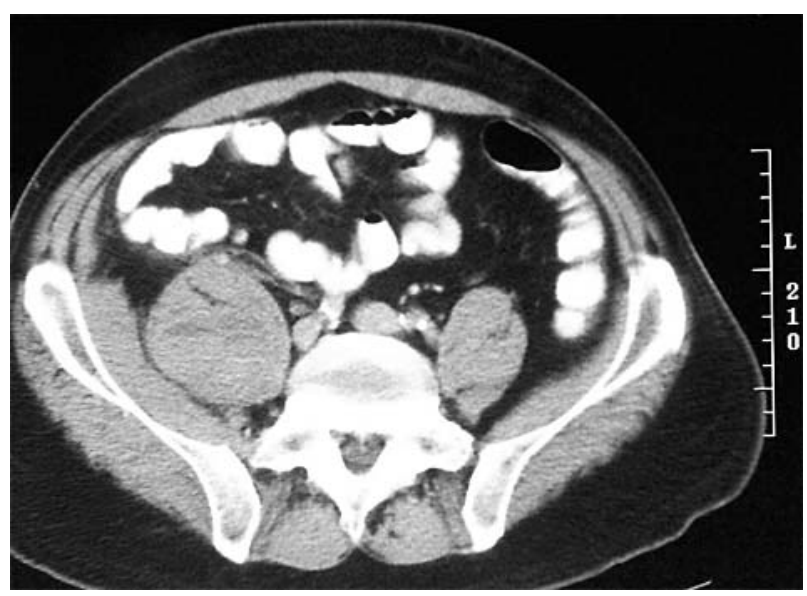

